\title{
La dieta iposodica riduce l'indice di sovrasaturazione urinaria dei sali di ossalato di calcio (AP-CaOx) nei pazienti con nefrolitiasi ossalato-calcica e sindrome metabolica
}

\author{
Francesca De Pascale, Michele Schiano di Cola, Riccardo Muscariello, Gianpaolo De Filippo, Pasquale Strazzullo, Domenico Rendina
}

Dipartimento di Medicina Clinica e Chirurgia, Università degli Studi di Napoli "Federico II", Napoli

\begin{abstract}
The hyposodic diet reduces urinary supersaturation index of calcium-oxalate salts in calcium-oxalate stone formers with metabolic syndrome

Background: Nutritional factors play a key role in the pathogenesis of both metabolic syndrome (MS) and calcium-oxalate $(\mathrm{CaOx})$ nephrolithiasis $(\mathrm{NL})$, two clinical entities strictly related. A reduction in dietary salt intake was universally recommended for treatment and prevention of MS and CaOx-NL. The aim of this study was to assess the efficacy of an hyposodic and normocalcic 7-days diet on the urinary supersaturation index of CaOx salts (AP ${ }_{\text {CaOx }}$ ), which is the driving force for CaOx crystallization and aggregation.

Patients and Methods: 18 patients with CaOx-NL and MS were evaluated on free diet and after 7 days of hyposodic and normocalcic metabolic diet. The adherence to prescribed metabolic diet was evaluated measuring the $24 \mathrm{~h}$ urinary sodium excretion before and after the diet: all enrolled patients showed an urinary sodium excretion $\leq 100 \mathrm{mmol} / 24 \mathrm{~h}$ after the diet.

Results: In addition to the decrease in urinary sodium excretion (185.2 \pm 9.9 vs $89.2 \pm 9.6 \mathrm{mmol} / 24 \mathrm{~h} ; \mathrm{p}<0.01)$, we observed also a reduction in the $24 \mathrm{~h}$ urinary calcium ( $6.49 \pm 0.09$ vs $5.31 \pm 0.25 \mathrm{mmol} / 24 \mathrm{~h} ; \mathrm{p}<0.01)$ and oxalate excretions $(0.44 \pm 0.06$ vs $0.30 \pm 0.04 \mathrm{mmol} / 24 \mathrm{~h} ; \mathrm{p}<0.01)$ and in the $24 \mathrm{~h}$ urinary volume $(1.85 \pm 0.09 \mathrm{vs} 1.61 \pm$ $0.09 \mathrm{mmol} / 24 \mathrm{~h} ; \mathrm{p}<0.01)$. $\mathrm{AP}_{\mathrm{CaOx}}$ declined significantly after the diet $(1.48 \pm 0.09 \mathrm{vs} 1.12 \pm 0.05 ; \mathrm{p}<0.01)$.

Discussion: The reduction in dietary salt intake significantly reduces the $\mathrm{AP}_{\mathrm{CaOx}}$ index and could play an important role in the prevention of $\mathrm{CaOx}-\mathrm{NL}$ associated to MS.
\end{abstract}

Keywords: APCaOx index, Metabolic syndrome, Nephrolithiasis, Salt, Sodium

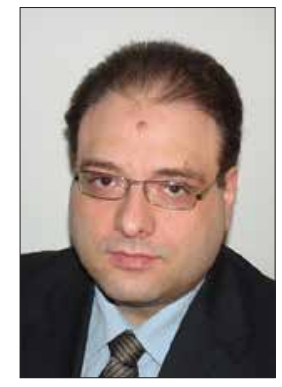

Domenico Rendina

\section{Introduzione}

La nefrolitiasi (NL) è una condizione clinica caratterizzata dalla formazione di composti cristallini (calcoli) nelle vie urinarie. Essa rappresenta una delle più frequenti modalità di presentazione delle patologie del metabolismo minerale e scheletrico (1). Nel corso degli ultimi anni, diversi studi epidemiologici e clinici, analiticamente esaminati in recenti

Accepted: February 6, 2017

Published online: February 23, 2017

Indirizzo per la corrispondenza:

Dr. Domenico Rendina

Dipartimento di Medicina Clinica e Chirurgia

Università degli Studi di Napoli "Federico II"

Via Sergio Pansini 5

80131 Napoli

strazzul@unina.it rassegne $(2,3)$, hanno evidenziato l'esistenza di uno stretto legame fisiopatologico esistente tra la NL e la sindrome metabolica (SM), uno dei principali fattori di rischio per patologie cardiovascolari (4). Sebbene siano stati proposti diversi criteri per la definizione diagnostica della SM, tutte le Società Scientifiche che si sono espresse sull'argomento concordano sulla necessità che per la diagnosi di SM sia presente l'associazione tra almeno tre di questi fattori: eccesso ponderale, alterazioni dell'omeostasi glucidica, disordini dell'omeostasi lipidica e ipertensione arteriosa (5). La NL da sali di calcio-ossalato ( $\mathrm{CaOx}$ ) è la forma più frequente di calcolosi renale osservata sia nella popolazione generale sia nei pazienti con $\operatorname{SM}(1,6)$. Questi ultimi presentano peculiari caratteristiche fenotipiche e cliniche a dieta libera (7). In particolare, i pazienti con SM e NL da CaOx presentano un'aumentata escrezione urinaria di sodio rispetto sia ai pazienti con NL e senza SM sia ai controlli sani senza SM e senza storia di NL (7). L'escrezione urinaria di sodio è considerata un indice attendibile dell'assunzione giornaliera di sale con la dieta (8). Lo studio epidemiologico di Taylor et al. ha dimostrato che l'aderenza a un regime iposodico, nell'ambito della dieta DASH (Dietary Approaches to 
Stop Hypertension), riduce significativamente l'incidenza di NL nella popolazione adulta (9). D'altra parte, le più recenti Linee Guida per la diagnosi, la prevenzione e il trattamento della nefrolitiasi $(10,11)$ nonché una recente revisione della letteratura internazionale sulla terapia dietetica della NL (12) sottolineano l'importanza di un regime dietetico iposodico e normocalcico nella prevenzione secondaria di questa malattia. Sulla base di queste premesse, scopo del presente lavoro è stato quello di valutare l'effetto di un breve periodo (7 giorni) di dieta iposodica e normocalcica sull'indice di sovrasaturazione urinaria dei sali di calcio-ossalato nei pazienti con SM e NL da CaOx.

\section{Materiali e metodi}

Tra i pazienti con NL afferenti all'Ambulatorio per lo studio delle malattie del metabolismo minerale e scheletrico del Dipartimento di Medicina Clinica e Chirurgia dell'Università degli Studi di Napoli "Federico II", sono stati considerati arruolabili quelli affetti da NL da $\mathrm{CaOx}$ recidivante (espulsione o rimozione chirurgica di 2 o più calcoli di ossalato di calcio) e con diagnosi clinica di SM posta in base ai criteri proposti dall'American Heart Association/National Heart, Lung, and Blood Institute per i soggetti caucasici (13). In particolare la diagnosi di SM è stata posta in pazienti che soddisfacevano almeno tre dei seguenti criteri clinici: (a) circonferenza vita $\geq 102 \mathrm{~cm}$ negli uomini $o \geq 88 \mathrm{~cm}$ nelle donne, (b) livelli sierici di trigliceridi $\geq 1.7 \mathrm{mmol} / \mathrm{L}$ a digiuno o trattamento farmacologico corrente per ipertrigliceridemia, (c) livelli sierici di colesterolo $\mathrm{HDL} \leq 1.03 \mathrm{mmol} / \mathrm{L}$ negli uomini o $\leq 1.3 \mathrm{mmol} / \mathrm{L}$ nelle donne o trattamento farmacologico in atto per bassi livelli di colesterolo HDL, (d) pressione arteriosa sistolica $\geq 130 \mathrm{mmHg}$ e/o pressione arteriosa diastolica $\geq 85 \mathrm{mmHg}$ o trattamento farmacologico antipertensivo in atto in un paziente con un'anamnesi personale positiva per ipertensione ed (e) glicemia a digiuno $\geq 5.6 \mathrm{mmol} / \mathrm{L}$ o trattamento farmacologico in atto per diabete (13). Tutti i soggetti arruolabili sono stati esaminati a dieta libera ed è stato successivamente loro prescritto un regime dietetico normocalorico (2200 kcal), iposodico (5 g/ die di sale) e normocalcico ( $1000 \mathrm{mg} /$ die di calcio) in cui era prevista l'assunzione di $900 \mathrm{mg} /$ die di fosforo, $300 \mathrm{~g} /$ die di carboidrati, $57 \mathrm{~g} /$ die di grassi e $70 \mathrm{~g} /$ die di proteine per la durata di 7 giorni. Alla fine del periodo di intervento dietetico, i pazienti sono stati rivalutati. Sono stati considerati aderenti alla dieta e, quindi, arruolati per lo studio i pazienti che evidenziavano un'escrezione urinaria di sodio $\leq 100 \mathrm{mmol} / 24 \mathrm{~h}$, corrispondente a un apporto alimentare quotidiano di sale < circa $6 \mathrm{~g}$. Sia a dieta libera che al termine dell'intervento dietetico sono state valutate le seguenti caratteristiche cliniche e biochimiche: altezza, peso, indice di massa corporea (IMC), pressione arteriosa (14), glicemia, trigliceridemia, HDL-colesterolemia, calcemia totale, fosfatemia, magnesemia, cloremia, sodiemia e potassiemia, PTH, $25 \mathrm{OHD}_{3}$, creatininemia ed esame delle urine a digiuno (tre ore dopo la prima minzione del mattino). Su una raccolta delle urine delle $24 \mathrm{~h}$ sono stati misurati $\mathrm{pH}$, creatinina, calcio, fosfato, sodio, potassio, cloro, magnesio, ossalato, citrato, acido urico e cistina.

$L^{\prime}$ indice $A P_{\text {caox }}$ è stato calcolato in base alla formula proposta e validata da Hess e Tiselius (15): $\mathrm{AP}_{\text {caox }}=\left(\mathrm{A} \times\right.$ calciuria $^{0.84} \times$ ossalaturia)/(citraturia ${ }^{0.22} \times$ magnesiuria ${ }^{0.12} \times$ volume $^{1.03}$ ), dove A è una costante determinata dalla lunghezza del periodo di raccolta delle urine.

L'analisi statistica è stata eseguita utilizzando il programma di calcolo SPSS-13. I dati sono espressi come media \pm SD e come valori assoluti (valore percentuale) per le variabili continue e discrete, rispettivamente. Il confronto dei valori pre e post dieta è stato eseguito utilizzando il T-test di Student per variabili appaiate. Un valore di $p<0.05$ è stato considerato statisticamente significativo.

\section{Risultati}

Le caratteristiche antropometriche dei pazienti arruolati e l'apporto dichiarato di macro-alimenti a dieta libera sono riportati nella Tabella I. Nella Tabella II sono riportati i parametri ematochimici misurati a dieta libera e dopo 7 giorni di regime dietetico iposodico: non sono state riscontrate differenze significative dei differenti parametri esaminati. Nella Tabella III sono riportati i parametri urinari misurati a dieta libera e dopo il regime dietetico iposodico e normocalcico. In aggiunta all'attesa e significativa riduzione dell'escrezione urinaria di sodio (185.2 \pm 9.9 vs $89.2 \pm 9.6 \mathrm{mmol} / 24 \mathrm{~h} ; \mathrm{p}<0.01 \mathrm{~T}$ di Student per variabili appaiate), al termine dell'intervento dietetico si osservano anche una significativa riduzione dell'escrezione urinaria di calcio (6.49 \pm 0.39 vs $5.31 \pm 0.25 \mathrm{mmol} / 24 \mathrm{~h} ; \mathrm{p}<0.01)$ e ossalato ( $0.44 \pm 0.06$ vs $0.30 \pm 0.04 \mathrm{mmol} / 24 \mathrm{~h} ; \mathrm{p}<0.01$ ) e una modesta ma significativa contrazione del volume urinario delle $24 \mathrm{~h}$ (1.85 \pm 0.09 vs $1617 \pm 0.09 \mathrm{mmol} / 24 \mathrm{~h} ; \mathrm{p}<0.01)$. Anche l'indice $\mathrm{AP}_{\text {caox }}$ risulta significativamente ridotto nei pazienti con $\mathrm{SM}$ dopo la dieta metabolica normocalcica e iposodica $(1.48 \pm 0.09$ vs $1.12 \pm 0.05 ; \mathrm{p}<0.01)$.

TABELLA I - Caratteristiche cliniche dei pazienti con NL da CaOx e SM a dieta libera

\begin{tabular}{lc}
\hline Numero & 18 \\
Maschi:Femmine & $9: 9$ \\
Età media (anni) & $45.1 \pm 3.7$ \\
Indice di Massa Corporea $\left(\mathrm{Kg} / \mathrm{m}^{2}\right)$ & $31.1 \pm 1.9$ \\
Età di esordio nefrolitiasi (anni) & $32.1 \pm 4.7$ \\
Episodi di nefrolitiasi (numero) & $7.1 \pm 2.4$
\end{tabular}

I dati sono espressi come media \pm deviazione standard per le variabili continue. NL da CaOx: nefrolitiasi da ossalato di calcio. SM: sindrome metabolica. La diagnosi clinica di SM è stata posta in base ai criteri proposti dall'American Heart Association/National Heart, Lung, and Blood Institute per i soggetti caucasici (13). 
TABELLA II - Parametri slerici dei pazienti con NL da CaOx e SM a dieta libera e dopo una settimana di dieta iposodica e normocalcica

\begin{tabular}{lccc}
\hline Parametri sierici & A dieta libera & Dopo la dieta & p-value \\
\hline Calcio totale (mmol/L) & $2.37 \pm 0.07$ & $2.37 \pm 0.07$ & 0.85 \\
Fosfati (mmol/L) & $1.09 \pm 0.11$ & $1.08 \pm 0.12$ & 0.45 \\
Magnesio (mmol/L) & $0.80 \pm 0.05$ & $0.81 \pm 0.06$ & 0.46 \\
Sodio (mmol/L) & $140.1 \pm 0.5$ & $139.9 \pm 0.7$ & 0.15 \\
Potassio (mmol/L) & $4.39 \pm 0.07$ & $4.41 \pm 0.06$ & 0.23 \\
PTH (pmol/L) & $4.50 \pm 0.51$ & $4.22 \pm 0.47$ & 0.09 \\
25OHD3 (nmol/L) & $72.9 \pm 19.4$ & $74.3 \pm 17.6$ & 0.17 \\
Creatinina (mmol/L) & $85.9 \pm 5.7$ & $82.1 \pm 6.1$ & 0.11 \\
\hline
\end{tabular}

I dati sono espressi come media \pm deviazione standard per le variabili continue. NL da CaOx: nefrolitiasi da ossalato di calcio. SM: sindrome metabolica. La diagnosi clinica di SM è stata posta in base ai criteri proposti dall'American Heart Association/National Heart, Lung, and Blood Institute per i soggetticaucasici (13). Composizione della dieta: $5 \mathrm{~g} /$ die di sale, $1000 \mathrm{mg} /$ die di calcio, $900 \mathrm{mg} /$ die di fosforo, $300 \mathrm{~g} /$ die di carboidrati, $57 \mathrm{~g} /$ die di grassi e $70 \mathrm{~g} /$ die di proteine $(2200 \mathrm{kcal} / \mathrm{die})$. II valore della p è determinato utilizzando il test $T$ di Student per variabili appaiate. Valori di $p<0.05$ sono considerati statisticamente significativi.

TABELLA III - Parametri urinari dei pazienti con NL da CaOx e SM a dieta libera e dopo una settimana di dieta iposodica e normocalcica

\begin{tabular}{lccc}
\hline Parametri urinari & A dieta libera & Dopo la dieta & p-value \\
\hline Sodiuria $(\mathrm{mmol} / 24 \mathrm{~h})$ & $185.2 \pm 9.9$ & $89.2 \pm 9.6$ & $<0.01$ \\
Volume $(\mathrm{L} / 24 \mathrm{~h})$ & $1.85 \pm 0.09$ & $1.61 \pm 0.09$ & $<0.01$ \\
Calciuria $(\mathrm{mmol} / 24 \mathrm{~h})$ & $6.49 \pm 0.39$ & $5.31 \pm 0.25$ & $<0.01$ \\
Citraturia $(\mathrm{mmol} / 24 \mathrm{~h})$ & $2.23 \pm 0.21$ & $2.19 \pm 0.16$ & 0.47 \\
Ossaluria $(\mathrm{mmol} / 24 \mathrm{~h})$ & $0.44 \pm 0.06$ & $0.30 \pm 0.04$ & 0.01 \\
Magnesiuria $(\mathrm{mmol} / 24 \mathrm{~h})$ & $3.48 \pm 0.15$ & $3.44 \pm 0.14$ & 0.09 \\
Uricuria $(\mathrm{mmol} / 24 \mathrm{~h})$ & $3.37 \pm 0.21$ & $3.01 \pm 0.21$ & 0.10 \\
APCaOx & $1.48 \pm 0.09$ & $1.12 \pm 0.05$ & $<0.01$
\end{tabular}

I dati sono espressi come media \pm deviazione standard per le variabili continue. NL da CaOx: nefrolitiasi da ossalato di calcio. SM: sindrome metabolica. APCaOx: indice di sovrasaturazione urinaria dei sali di calcio ossalato (15) La diagnosi clinica di SM è stata posta in base ai criteri proposti dall'American Heart Association/National Heart, Lung, and Blood Institute per i soggetticaucasici (13). Composizione della dieta: $5 \mathrm{~g} /$ die di sale, $1000 \mathrm{mg} /$ die di calcio, $900 \mathrm{mg} /$ die di fosforo, $300 \mathrm{~g} /$ die di carboidrati, $57 \mathrm{~g} /$ die di grassi e $70 \mathrm{~g} /$ die di proteine $(2200 \mathrm{kcal} / \mathrm{die})$. II valore della p è determinato utilizzando il test $T$ di Student per variabili appaiate. Valori di $p<0.05$ sono considerati statisticamente significativi.

\section{Discussione}

I risultati dello studio forniscono in primis una conferma del fondamentale ruolo svolto dai fattori nutrizionali nella patogenesi della NL da CaOx in pazienti con SM. I dati epidemiologici disponibili certificano che negli ultimi decenni dello scorso secolo si è verificato un aumento significativo dell'incidenza e della prevalenza della NL e della SM nel mondo, in particolare nei paesi industrializzati (16). Nel caso della NL è stato anche riscontrato un significativo patomorfismo della patologia (17). La stessa tipologia di dati suggerisce che, alla base di questi cambiamenti epidemiologici, che riguardano non solo la NL e la SM, ma, in generale, tutte le patologie mediche multifattoriali, vi sia un radicale cambiamento delle abitudini alimentari. In Italia e nei paesi del bacino del Mediterraneo questo cambiamento è caratterizzato da un progressivo allontanamento dalla dieta mediterranea a favore di una dieta simile a quella americana da "fast food", ricca di grassi insaturi $(18,19)$. In aggiunta, i risultati dello studio confermano che il cambiamento delle abitudini alimentari in pazienti con SM e NL da CaOx è un presupposto indispensabile per una corretta prevenzione secondaria della NL, una patologia che, se non adeguatamente trattata, è caratterizzata da un tasso di recidiva superiore al $50 \%$ in un lustro (20). L'escrezione urinaria di sodio è un indice attendibile dell'introito alimentare di sale (8). L'introito alimentare di sale e la conseguente escrezione urinaria di sodio influenzano significativamente l'assorbimento intestinale e l'escrezione renale di calcio e di ossalato, due potenti fattori litogenici. Un aumentato apporto alimentare di sale può infatti favorire la formazione nel lume intestinale di ossalato di sodio, un sale facilmente assorbibile a livello intestinale. Si verifica in questo modo un aumento del carico renale di ossalato e dell'ossalaturia. In parallelo, il carico maggiore di sodio che è filtrato a livello glomerulare arriva al tubulo renale e diminuisce il riassorbimento tubulare del calcio, con conseguente aumento dell'escrezione urinaria di calcio $(20,21)$. L'aumento dell'escrezione urinaria di calcio e di ossalati concorre ad aumentare significativamente l'indice $\mathrm{AP}_{\text {Caox }}$, che rappresenta una misura elettrochimica della litogenicità urinaria.

\section{Conclusioni}

Le abitudini alimentari giocano un ruolo centrale nella patogenesi della NL associata a SM. L'insieme dei dati conferma che opportune modifiche del comportamento alimentare, con strategie sanitarie proponibili su larga scala (riduzione del consumo alimentare di sodio), possono avere effetti significativi in termini di prevenzione.

\section{Disclosures}

Financial support: No financial support was received for this submission. Conflict of interest: The authors have no conflict of interest.

Studi sperimentali su esseri umani: Approvazione ottenuta dal Comitato Etico. 


\section{Bibliografia}

1. Favus MJ. Clinical approach to metabolic bone disease. In: DeGroot LJ, Jameson JL edt, Endocrinology. W.B. Sauders Company, Philadelphia, $4^{\circ}$ edition 2001:1181-1186.

2. Rendina D, De Filippo G, D’Elia L, Strazzullo P. Metabolic syndrome and nephrolithiasis: a systematic review and meta-analysis of the scientific evidence. J Nephrol. 2014;27:371-376.

3. Rendina D, De Filippo G, Zampa G, Muscariello R, Mossetti G, Strazzullo P. Revisione critica della letteratura internazionale di pertinenza internistica prodotta nel biennio 2007-2008 sul tema della nefrolitiasi. Giornale di Tecniche Nefrologiche e Dialitiche. 2009;21:49-52.

4. Grundy SM. Metabolic syndrome update. Trends Cardiovasc Med. 2016;26:364-373.

5. Strazzullo $P$, Barbato A, Siani A, et al. Diagnostic criteria for metabolic syndrome: a comparative analysis in an unselected sample of adult male population. Metabolism. 2008;57: 355-361.

6. Kadlec AO, Greco K, Fridirici ZC, Hart ST, Vellos T, Turk TM. Metabolic syndrome and urinary stone composition: what factors matter most? Urology. 2012; 80:805-810.

7. De Pascale F, Muscariello R, Zampa G, De Filippo G, Rendina D, Strazzullo P. Caratteristiche cliniche dei pazienti con sindrome metabolica e nefrolitiasi recidivante da ossalato di calcio. Giornale di Tecniche Nefrologiche e Dialitiche. 2012;24:19-23.

8. Cappuccio FP, Ji C, Donfrancesco C, et al. Geographic and socioeconomic variation of sodium and potassium intake in Italy: results from the MINISAL-GIRCSI programme. BMJ Open. 2015; 5:e007467.

9. Taylor EN, Fung TT, Curhan GC. DASH-style diet associates with reduced risk for kidney stones. J Am Soc Nephrol. 2009; 20:2253-2259.

10. http://uroweb.org/wp-content/uploads/22-Urolithiasis_LR_ full.pdf. Accesso Gennaio 30, 2017.

11. Gambaro G, Croppi E, Coe F, et al. Metabolic diagnosis and medical prevention of calcium nephrolithiasis and its systemic manifestations: a consensus statement. J Nephrol. 2016; 29:715-734.

12. Prezioso D, Strazzullo $P$, Lotti $T$, et al. Dietary treatment of urinary risk factors for renal stone formation. A review of CLU Working Group. Arch Ital Urol Androl. 2015;87:105-120.

13. Grundy SM, Cleeman JI, Daniels SR, et al. Diagnosis and management of the metabolic syndrome: an American Heart Association/National Heart, Lung, and Blood Institute Scientific Statement. Circulation. 2005;112:2735-2752.

14. http://siia.it/wp-content/uploads/2011/06/linee-guida-sullamisurazione-convenzionale-e-auto-243.pdf. Accesso Gennaio 30, 2017.

15. Hess B, Tiselius HG. Physical-chemical process in kidney stone formation. In: Coe FL, Favus MJ eds. Disorders of Bone and Mineral Metabolism Lippincot Williams \& Wilkins, Philadelphia 2002:619-628.

16. Kopple JD. Obesity and chronic kidney disease. J Ren Nutr. 2010; 20:S29-30.

17. Rendina D, De Filippo G, De Pascale F, et al. The changing profile of patients with calcium nephrolithiasis and the ascendancy of overweight and obesity: a comparison of two patient series observed 25 years apart. Nephrol Dial Transplant. 2013;28(Suppl. 4):iv146-151.

18. Silva R, Bach-Faig A, Raidó Quintana B, et al. Worldwide variation of adherence to the Mediterranean diet, in 1961-1965 and 2000-2003. Public Health Nutr. 2009;12:1676-1684.

19. Leclercq C, Arcella D, Piccinelli R, et al. The Italian National Food Consumption Survey INRAN-SCAI 2005-06: main results in terms of food consumption. Public Health Nutr. 2009;12: 2504-2532.

20. Coe FL, Evan A, Worcester E. Kidney stone disease. J Clin Invest. 2005;115:2598-2608.

21. Taylor EN, Curhan GC. Determinants of 24-hour urinary oxalate excretion. Clin J Am Soc Nephrol. 2008;3:1453-1460. 